\title{
Temperature Dependence of Ultrafast Phonon Dynamics in Graphite
}

M. Scheuch, ${ }^{1,2}$ T. Kampfrath, ${ }^{3}$ M. Wolf, ${ }^{3}$ K. von Volkmann, ${ }^{3}$ C. Frischkorn, ${ }^{4}$ and L. Perfetti ${ }^{4}$

1) Fritz-Haber-Institut der Max-Planck-Gesellschaft, Berlin, Germany

2) Fachbereich Physik, Freie Universität Berlin, Germany

3) APE Angewandte Physik und Elektronik GmbH, Berlin, Germany

${ }^{4)}$ Laboratoire des Solides Irradiés, Ecole polytechnique, Palaiseau cedex, France

(Dated: 4 November 2011)

Nonequilibrium optical phonons are generated in graphite following the excitation of electron-hole pairs with a femtosecond laser pulse. Their energy relaxation is probed by means of terahertz pulses. We find that the hot-phonon lifetime increases by a factor of 2 when the sample temperature decreases from 300 to $5 \mathrm{~K}$. These results suggest that the energy relaxation in graphite at room temperature and above is dominated by the anharmonic decay of hot $A_{1}^{\prime}$ phonons at the $\mathrm{K}$ point into acoustic phonons with energies of about $10 \mathrm{meV}$.

In recent years, graphene and graphite have earned great interest due to their ability to sustain high current densities ${ }^{14}$ For this reason, carbon-based materials are considered as promising alternatives to silicon for the development of nanoelectronic devices. Transport experiments in graphite and carbon nanotubes have revealed a critical driving electric field at which a crossover from ballistic to diffusive transport occurs. This crossover was assigned to the scattering of high-energy electrons with a minor subset of strongly coupled optical phonons (SCOPs), which acquire a higher effective temperature than the remaining, still cold phonons ${ }^{5}$. Density functional theory (DFT) calculations of electron-phonon coupling have identified the SCOPs in graphite as the modes with the highest energies $(\approx 0.2 \mathrm{eV})$ at the $\Gamma$ point $\left(E_{2 g}\right)$ and the $\mathrm{K}$ point $\left(A_{1}^{\prime}\right)$ of the Brillouin zone 6 6/7

Hot SCOPs can also be generated by illuminating graphite with a femtosecond laser pulse ${ }^{8} 20$ The photoexcited electrons thermalize and concurrently transfer most of their excess energy to the small phonon subset of SCOPs within $0.5 \mathrm{ps} 9 \frac{9}{1621}$ A quasi-equilibrium between electrons and SCOPs is established. The hot phonons cool on a picosecond timescale by energy transfer to the other, cold phonons. Up to now, however, the pathway of the hot-phonon relaxation has not yet been studied in detail. Theory work predicts that the SCOP cooling proceeds via anharmonic decay, which becomes significantly faster with increasing ambient temperature ${ }^{22}$ Such behavior has already been indicated by time-resolved Raman spectroscopy at temperatures from 300 to $700 \mathrm{~K} ! 13$ In order to elucidate the role of decay channels involving low-energy phonons, experiments have to be performed at lower temperatures as well.

In this Letter, we make use of time-resolved $\mathrm{THz}$ spectroscopy to measure the energy decay rate of the hotphonon system in photoexcited, highly ordered pyrolytic graphite (HOPG) as a function of temperature from 5 to $300 \mathrm{~K}$. Due to its low photon energy, THz radiation is particularly sensitive to the distribution of charge carriers in the vicinity of the Fermi energy ${ }^{[2]}$ We observe a pronounced increase of the SCOP lifetime with decreasing temperature. This result is consistent with a dominant SCOP decay into acoustic modes with energies of only $10 \mathrm{meV}$ via anharmonic coupling.
We apply 12 -fs laser pulses with 780 -nm center wavelength from an $80-\mathrm{MHz}$ Ti:sapphire oscillator to generate $\mathrm{THz}$ pulses via difference frequency mixing in a GaSe crystal $^{24}$ The resulting $\mathrm{THz}$ pulses cover a range from 10 to $30 \mathrm{THz}$ with a duration of $100 \mathrm{fs}$. The detection of the THz electric field via electro-optic sampling in ZnTe allows us to simultaneously measure both amplitude and phase of the $\mathrm{THz}$ transient ${ }^{25}$ Part of the oscillator output is used to excite the sample prior to $\mathrm{THz}$ probing at variable delay times $\tau$. The samples are prepared by peeling off flakes from a HOPG crystal, resulting in a 20$\mathrm{nm}$ thin film supported on a diamond substrate which is mounted in a cryostat that can be cooled down to $5 \mathrm{~K}$. Transmitting under normal incidence, the $\mathrm{THz}$ pulse probes the optical properties perpendicular to the $c$ axis. In our setup, we measure the electric fields $E_{0}(t)$ and $E_{0}(t)+\Delta E_{\tau}(t)$ of a $\mathrm{THz}$ pulse that has traversed an unexcited and excited sample, respectively. Here, $t$ denotes the time axis of the $\mathrm{THz}$ transient.

Figure11(a) shows the pump-induced changes $\Delta E_{\tau}(t)$ in the transmitted $\mathrm{THz}$ field along with the reference field $E_{0}(t)$. In order to monitor the relaxation of the excited sample, we set $t=t_{\max }$, where the maximum of the pump-induced $\mathrm{THz}$ transient is located. The resulting trace of $\Delta E_{\tau}\left(t_{\max }\right)$ vs. pump-probe delay $\tau$ is shown in Fig.1.(b) for two pump fluences. All traces exhibit a biexponential decay with a fast and slow component featuring time constants of $0.8 \mathrm{ps}$ and $5.5 \mathrm{ps}$ at $300 \mathrm{~K}$, respectively. As indicated by the identical slopes of the curves in Fig.1.(b), the slow time constants are found to be independent of the applied laser fluence demonstrating that they represent sample-intrinsic quantities.

Qualitatively similar two-component dynamics have been observed using other time-resolved techniques $\frac{8111-18}{18}$ Further analysis ${ }^{9 / 12}$ and simulations based on Boltzmann Peierls rate equations ${ }^{21}$ have shown that the faster signal component reflects the thermalization of the electronic subsystem via electron-electron and electron-phonon scattering accompanied by energy transfer to the SCOPs. Since the Fermi surface of graphite consists of only two small pockets, the emitted phonons have a wavevector whose in-plane component is located close to the $\Gamma$ or K point of the Brillouin zone ${ }^{9}$ Despite the small fraction of available phonon states, the 


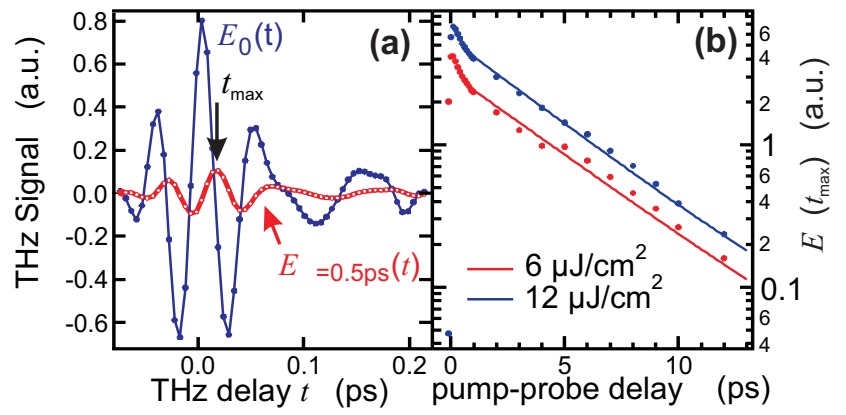

FIG. 1. (a) THz waveform $E_{0}(t)$ after transmission through unexcited graphite (blue) and pump-induced changes $\Delta E_{\tau}(t)$ at $\tau=0.5 \mathrm{ps}$ (red). Black arrow: pump-induced signal maximum $\Delta E_{\tau}\left(t_{\max }\right)$. (b) $\Delta E_{\tau}\left(t_{\max }\right)$ as function of pump-probe delay at $300 \mathrm{~K}$ at various pump fluences. Solid lines: biexponential fit.

remarkably fast electron cooling is brought about by the strong electron-phonon coupling in graphite $\frac{617}{}$ After less than $0.5 \mathrm{ps}$, the SCOPs have absorbed more than $90 \%$ of the electronic excess energy, and a quasi-equilibrium of electrons and SCOPs is established ${ }^{9}[12$ The slower component of the pump-probe signal is associated with the cooling of the combined subsystem electrons plus hot SCOPs $\frac{9110}{9}$ Since most of the pump energy is contained in the hot phonons, the slow decay directly reflects the SCOP lifetime. 10121

In order to obtain deeper insights into the energy decay of the SCOPs, we took pump-probe traces $\Delta E_{\tau}\left(t_{\max }\right)$ at various ambient temperatures $T_{\mathrm{a}}$. As shown in Fig.2(a), the decay of the slower component becomes faster with rising $T_{\mathrm{a}}$. We obtain the hot SCOP lifetimes as the time constant $1 / \gamma$ of the slower relaxation component of the signal. Fig. 2(b) shows the resulting decay rate $\gamma$ vs. $T_{\mathrm{a}}$. Note that $\gamma$ increases by more than $100 \%$ when the ambient temperature increases from 5 to $300 \mathrm{~K}$.

The cooling of the electron-SCOP system proceeds via annihilation of an electron-hole pair ${ }^{[26}$ or a SCOP ${ }^{[22}$ both resulting in phonon emission into cold lattice modes. The first scenario is not expected to occur on the picosecond time scale observed in our experiment as it involves emitted phonons with energies more than one order of magnitude smaller than those of the SCOPs $\frac{10}{10}$ In addition, the coupling of these phonons to the electrons is negligible as compared to the SCOPs. ${ }^{[6}$ Finally, according to the two-temperature model ${ }^{[26}$ the first mechanism should result in a slower decay with increasing $T_{\mathrm{a}}$, contrary to our findings [Fig. 2(b)]. Thus, it is reasonable to assume the combined cooling of electrons and hot SCOPs mediated by anharmonic phonon-phonon coupling which induces the decay of a SCOP into two phonons with

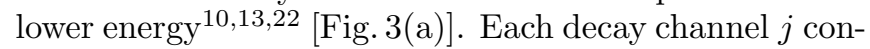
tributes

$$
\gamma_{j}=\gamma_{j 0}\left[1+b\left(\omega_{j}^{\prime}, T_{\mathrm{a}}\right)+b\left(\omega_{j}-\omega_{j}^{\prime}, T_{\mathrm{a}}\right)\right]
$$

to the total energy decay rate $\gamma=\sum \gamma_{j}$ of the hotphonon system. ${ }^{27}$ Here, $\gamma_{j 0}$ is the rate at vanishing sam-
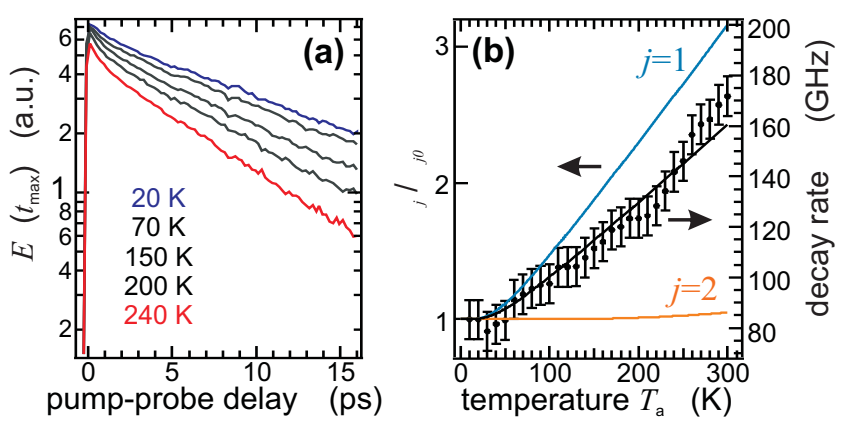

FIG. 2. (a) Decay of $\Delta E_{\tau}\left(t_{\max }\right)$ vs. $\tau$ at several temperatures. (b) $T_{\mathrm{a}}$ dependence of the resulting scattering rates $\gamma$ (right axis). Solid lines: normalized contributions (left axis) of phonon decay channels $j=1,2$ shown in Fig. 3(b) and fit of $\gamma_{1}+\gamma_{2}$ to the experimentally determined $\gamma$.

ple temperature, $b$ the Bose-Einstein distribution, $\omega_{j}$ the SCOP frequency, and $\omega_{j}^{\prime}$ and $\omega_{j}-\omega_{j}^{\prime}$ are the frequencies of the emitted phonons. Each of these three-phonon processes has to conserve phonon energy and wavevector, thus greatly reducing the set of allowed final modes. Using the phonon dispersion relation of graphite, an experimentally determined function,, 7 it has been found ${ }^{22}$ that the lower phonon energy $\hbar \omega_{j}^{\prime}$ can only derive from a few narrow intervals between 0 and $\hbar \omega_{j} / 2$. Examples of decay events are shown in Fig. 3(b).

The interval with the lowest phonon energies is centered around $\hbar \omega_{1}^{\prime}=10.5 \mathrm{meV}$ and associated with acoustic phonons emitted in the decay of the $A_{1}^{\prime}-\mathrm{K}$ mode ${ }^{22}$ [blue arrows in Fig. 3(b)]. Using Eq. (1), we calculate the normalized rate $\gamma_{1} / \gamma_{10}$ of this decay channel as a function of $T_{\mathrm{a}}$. As seen from Fig.2( $\left.\mathrm{b}\right), \gamma_{1}$ increases by more than a factor of 2 when the temperature increases from 5 to $300 \mathrm{~K}$. This strong temperature sensitivity arises because the phonon energy $\hbar \omega_{1}^{\prime}$ is comparable to the thermal energy $k_{\mathrm{B}} T_{\mathrm{a}}$ [light red area in Fig.3(b)]. In contrast, considering an allowed decay channel with the next-higher energy $\hbar \omega_{2}^{\prime}=60 \mathrm{meV}$ of the emitted lowenergy phonon ${ }^{22}$ [orange arrows in Fig. 33(b)], we obtain a curve with nearly negligible temperature dependence $[j=2$ curve in Fig. 2(b)]. Similar curves are obtained for all remaining decay channels $j$ as they involve even higher energies $\hbar \omega_{j}^{\prime}$. An example is the symmetric decay of the $E_{2 g}-\Gamma$ mode [red arrows in Fig.33(b)]. Therefore, it is sufficient to fit a linear combination of $\gamma_{1}$ and $\gamma_{2}$ to the measured decay curve of $\gamma$ vs. $T_{\mathrm{a}}$, with $\gamma_{10}$ and $\gamma_{20}$ as fit parameters. Whereas $\gamma_{2}$ merely contributes a constant offset, $\gamma_{1}$ sets the slope of the curve. Best fit results are obtained for $1 / \gamma_{10}=22.1 \mathrm{ps}$ and $1 / \gamma_{20}=30.5 \mathrm{ps}$ [Fig.2(b)]. Thus, our results are compatible with the notion that the energy relaxation of the hot SCOPs is dominated by the decay of $A_{1}^{\prime}$ phonons at the $\mathrm{K}$ point into acoustic phonons with energies of about $10 \mathrm{meV}$.

Previous temperature-dependent studies of the SCOP decay of photoexcited graphite made use of time-resolved incoherent Raman scattering and report decay times of $2.2 \mathrm{ps}$ and $2.4 \mathrm{ps} \$^{17}$ at $300 \mathrm{~K}$, considerably shorter 


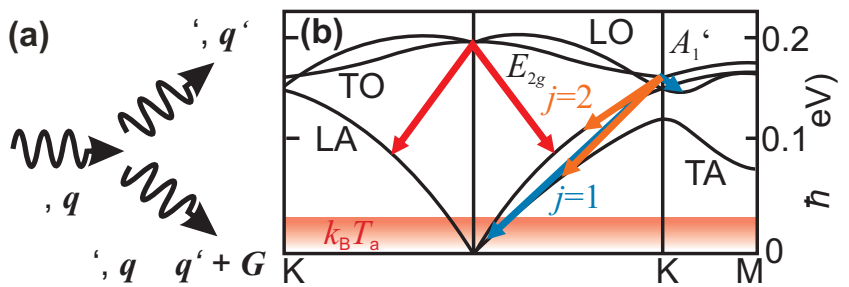

FIG. 3. (a) Schematic of phonon decay into two phonons. Wavevector $\boldsymbol{q}$ and energy $\hbar \omega$ conservation restricts the set of allowed decay channels. $\boldsymbol{G}$ is a reciprocal lattice vector. (b) Schematic of graphite phonon dispersion. Arrows: examples of allowed decay channels of SCOPs according to Ref.6. Orange area indicates the thermal energy $k_{\mathrm{B}} T_{\mathrm{a}}$ with $T_{\mathrm{a}} \leq 300 \mathrm{~K}$.

than the 5.5 ps observed here. It should be noted that studies based on other time-resolved techniques such as transient reflectance or transmittance, $\frac{818}{18}$ photoelectron spectroscopy, ${ }^{12}$ and electron diffraction ${ }^{16}$ also report a broad variety of time constants of the slow signal component. This variety may be related to the use of different probing techniques and samples of different thickness. $\frac{1820}{2 s p e c i a l l y, ~ s i n c e ~ b o t h ~} \mathrm{TH}_{z}{ }^{9}$ and Raman signa $\sqrt{10}$ reflect the hot-phonon temperature, the different time constants measured most likely arise from the samples used. In this work, the graphite thin film is homogeneously excited, whereas transport effects into the inhomogeneously excited bulk crystal used by Ref. 13 may accelerate the dynamics inside the probed volume. However, despite the different time constants, the relative changes as a function of $T_{\mathrm{a}}$ are comparable for the Raman-based and our work: an increase of the phonon decay rate by about $70 \%$ was observed by increasing $T_{\mathrm{a}}$ from 300 to $700 \mathrm{~K}$ (Refs. 13 and 17) while a $100 \%$ increase from 5 to $300 \mathrm{~K}$ is found in our experiment. We also note that our fit to the measured $\gamma\left(T_{\mathrm{a}}\right) / \gamma(300 \mathrm{~K})$ would also give a reasonable description of the normalized time-resolved Raman data. Conversely, the model of Ref. 13 would not yield a good description of our data as it involves too high phonon energies $\hbar \omega_{j}^{\prime}$. In other words, low-temperature experiments allow a better identification of the phonon decay processes involving small phonon energies of the order of $10 \mathrm{meV}$.

We finally compare our results to DFT calculations $\frac{22 \mid 28}{2}$ of the lifetime of various phonon modes at the $\Gamma$ and $K$ points. Our results agree well with the predicted temperature dependence of the $A_{1}^{\prime}$-K lifetime [see Fig. 4(a) in Ref. 22] as well as the branching ratio of the different decay processes $j$ : we have $\gamma_{01} / \sum \gamma_{0 j}=0.58$ as inferred from our experiment and 0.56 from theory. Absolute numbers agree roughly: $1 / \gamma_{10}=22.1 \mathrm{ps}$, as derived from experiment, is larger than $1 / \gamma_{10}=8.2 \mathrm{ps} / 0.56=$ $14.6 \mathrm{ps}$ predicted by theory. However, theory ${ }^{22}$ also suggests a strong contribution from the decay of the $E_{2 g}-\Gamma$ mode, with a much shorter lifetime of about $3.3 \mathrm{ps}$ at vanishing temperature. Such short phonon lifetimes are not observed in our experiment. The reason for this dis- crepancy is not yet understood.

In conclusion, we have observed a pronounced acceleration of the hot-phonon decay in graphite in the temperature range when temperature is increased from 5 to $300 \mathrm{~K}$. Our results suggest that this relaxation proceeds via anharmonic decay of hot SCOPs into acoustic phonons with low energy. As a consequence, the hotphonon population observed in high-field transport in graphite and graphene ${ }^{4}$ should roughly scale with the inverse of the ambient temperature. This notion would imply an increased resistance and a lower breakdown threshold for cooled graphite-based devices.

The authors would like to thank the German Research Foundation DFG for financial support via Sfb 450.

${ }^{1}$ K. S. Novoselov, A. K. Geim, S. V. Morozov, D. Jiang, Y. Zhang, S. V. Dubonos, I. V. Grigorieva, and A. A. Firsov, Science 306, $666(2004)$

${ }^{2}$ J. Moser, A. Barreiro, and A. Bachtold, Appl. Phys. Lett. 91, 163513 (2007)

${ }^{3}$ A. Barreiro, M. Lazzeri, J. Moser, F. Mauri, and A. Bachtold, Phys. Rev. Lett. 103, 076601 (2009)

${ }^{4}$ S. Berciaud, M. Y. Han, K. F. Mak, L. E. Brus, P. Kim, and T. F. Heinz, Phys. Rev. Lett. 104, 227401 (2010)

${ }^{5}$ A. Javey, J. Guo, M. Paulsson, Q. Wang, D. Mann, M. Lundstrom, and H. Dai, Phys. Rev. Lett. 92, 106804 (2004)

${ }^{6}$ S. Piscanec, M. Lazzeri, F. Mauri, A. C. Ferrari, and J. Robertson, Phys. Rev. Lett. 93, 185503 (2004)

${ }^{7}$ J. Maultzsch, S. Reich, C. Thomsen, H. Requardt, and P. Ordejón, Phys. Rev. Lett. 92, 075501 (2004)

${ }^{8}$ H. Wang et al., Appl. Phys. Lett. 96, 081917 (2010).

${ }^{9}$ T. Kampfrath, L. Perfetti, F. Schapper, C. Frischkorn, and M. Wolf, Phys. Rev. Lett. 95, 187403 (2005)

${ }^{10}$ H. Yan, D. Song, K. F. Mak, I. Chatzakis, J. Maultzsch, and T. F. Heinz, Phys. Rev. B 80, 121403(R) (2009)

${ }^{11}$ M. Breusing, C. Ropers, and T. Elsaesser, Phys. Rev. Lett. 102, 086809 (2009)

${ }^{12}$ Y. Ishida, T. Togashi, K. Yamamoto, M. Tanaka, T. Taniuchi, T. Kiss, M. Nakajima, T. Suemoto, and S. Shin, Sci. Rep. 1, 64 (2011).

${ }^{13}$ I. Chatzakis, H. Yan, D. Song, S. Berciaud, and T. F. Heinz, Phys. Rev. B 83, 205411 (2011)

${ }^{14}$ G. Moos, C. Gahl, R. Fasel, M. Wolf, and T. Hertel, Phys. Rev. Lett. 87, 267402 (2001)

${ }^{15}$ K. Ishioka, M. Hase, M. Kitajima, L. Wirtz, A. Rubio, and H. Petek, Phys. Rev. B 77, 121402(R) (2008)

${ }^{16}$ S. Schäfer, W. Liang, and A. H. Zewail, New J. Phys. 13 (2011) 063030

${ }^{17}$ K. Kang, D. Abdula, D. G. Cahill, and M. Shim, Phys. Rev. B 81, 165405 (2010)

${ }^{18}$ R. W. Newson, J. Dean, B. Schmidt, and H. M. van Driel, Optics Express 17, No. 4, 2326 (2009)

${ }^{19}$ H. Choi, F. Borondics, D. A. Siegel, S. Y. Zhou, M. C. Martin, A. Lanzara, and R. A. Kaindl, Appl. Phys. Lett. 94, 172102 (2009)

${ }^{20}$ P. J. Hale, S. M. Hornett, J. Moger, D. W. Horsell, and E. Hendry, Phys. Rev. B 83, 121404(R) (2011)

${ }^{21}$ S. Butscher, F. Milde, M. Hirtschulz, E. Malic, and A. Knorr, Appl. Phys. Lett. 91, 203103 (2007)

${ }^{22}$ N. Bonini, M. Lazzeri, N. Marzani, and F. Mauri, Phys. Rev. Lett. 99, 176802 (2007)

${ }^{23}$ R. Ulbricht, E. Hendry, J. Shan, T. F. Heinz, and M. Bonn, Rev. Mod. Phys. 83, 543 (2011)

${ }^{24}$ R. Huber, A. Brodschelm, F. Tauser, and R. Leitenstorfer, Appl. Phys. Lett. 76, 3191 (2000)

${ }^{25}$ T. Kampfrath, J. Nötzold, and M. Wolf, Appl. Phys. Lett. 90, 231113 (2007)

${ }^{26}$ P. B. Allen, Phys. Rev. Lett. 59, 1460 (1987) 
${ }^{27}$ A. Laubereau, D. von der Linde, and W. Kaiser, Phys. Rev. Lett. 27, 802 (1971)

${ }^{28}$ N. Bonini, R. Rao, A. M. Rao, M. Marzari, and J. Menéndez, phys. stat. sol. (b) 245, No. 10, 2149-2154 (2008) 